\title{
PREVISÃO DE RADIAÇÃO SOLAR INCIDENTE NO ESTADO DO CEARÁ - BRASIL
}

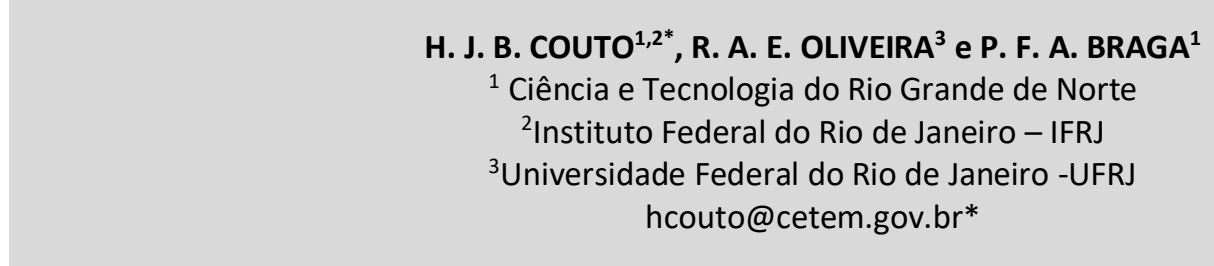

Artigo submetido em janeiro/2015 e aceito em setembro/2016

DOI: $10.15628 /$ holos. 2016.2706

\section{RESUMO}

Esse trabalho tem como objetivo fazer previsão de radiação solar para geração de energia elétrica no Estado do Ceará, nordeste Brasileiro. Para isso, foi realizado comparação de métodos estatísticos de previsão (médias móveis e projeção exponencial) para geração de séries temporais, aplicados a radiação solar incidente, cujos dados reais foram medidos nas dependências do Departamento de Engenharia Elétrica (DEE) da Universidade Federal do Ceará (UFC), campus Fortaleza, de propriedade do Laboratorio de Energias Alternativas -
LEA da UFC . Com essa pesquisa, ficou constatado que o método de projeção exponencial é a melhor escolha para se realizar uma previsão de série temporal a partir dos dados mencionados. Essa conclusão é baseada no fato de que este método tanto para o período chuvoso, como para o período seco, fornece os menores valores de RMSE como, por exemplo, para o mês de outubro no horário de $17: 00 \mathrm{~h}$ temos $1,40 \mathrm{~W} / \mathrm{m}^{2}$, e para o MAPE, temos o valor de $4,48 \%$ também para o mês de outubro as $13: 00 h$.

PALAVRAS-CHAVE: energia solar, previsão, estado do Ceará.

\section{FORECASTING SOLAR RADIATION INCIDENT IN CEARÁ STATE - BRAZIL}

\begin{abstract}
This study aims to forecast solar radiation to electricity generation in the state of Ceara, northeastern Brazil. For this, we performed comparative statistical forecasting methods (moving average and exponential projection) to generate time series, applied to incoming solar radiation, whose real data were measured in the Department of Electrical Engineering (DEE) of the Federal University of Ceará (UFC), Fortaleza campus, owned by the Laboratory of Alternative Energy - LEA UFC. With this research, it was
\end{abstract}

found that the exponential projection method is the best choice to perform a time-series forecast from the data mentioned. This conclusion is based on the fact that this method for both the rainy season, as the dry period, provides the smallest RMSE values as, for example, for the month of October in 17 hours: 00 have $1.40 \mathrm{~W} / \mathrm{m}^{2}$, and the MAPE, we have the value of $4.48 \%$ also for the month of October the 13: 00h.

KEYWORDS: solar radiation, forecasting, Ceará state. 


\section{INTRODUÇÃO}

O tema "Energias Renováveis" encontra-se em bastante evidência em tempos atuais. Isso pode ser justificado pelo consenso mundial da comunidade acadêmica no sentido de alertar, cada vez mais, para os perigos que podemos enfrentar com o aumento acelerado do efeito estufa, oriundo de processos de combustão, devido à utilização de fontes energéticas extremamente poluentes, como é o caso do petróleo e também com o funcionamento de termelétricas que no seu processo de conversão de energia elétrica, utiliza a queima do carvão mineral. Para que seja possível pensar em um desenvolvimeto sustentável, investimentos em geração de energias renováveis devem ser feitos cada vez mais, como por exemplo, em energia solar e eólica.

O nordeste brasileiro é bastante abundante em energia solar, isso é o que afirma o Atlas Solarimétrico Brasileiro (TIBA, C. et al, 2000). Por exemplo, a radiação solar global diária, média anual no Ceará chega a um valor de $20 \mathrm{MJ} / \mathrm{m}^{2}$.dia como ilustrado na Fig. (1), que por sua vez, quando comparado com outras regiões pelo mundo a fora, por exemplo, Dongola - Sudão (região extremamente seca, quase o ano todo), é possível encontrarmos valores da ordem de 23,8 $\mathrm{MJ} / \mathrm{m}^{2}$. dia.

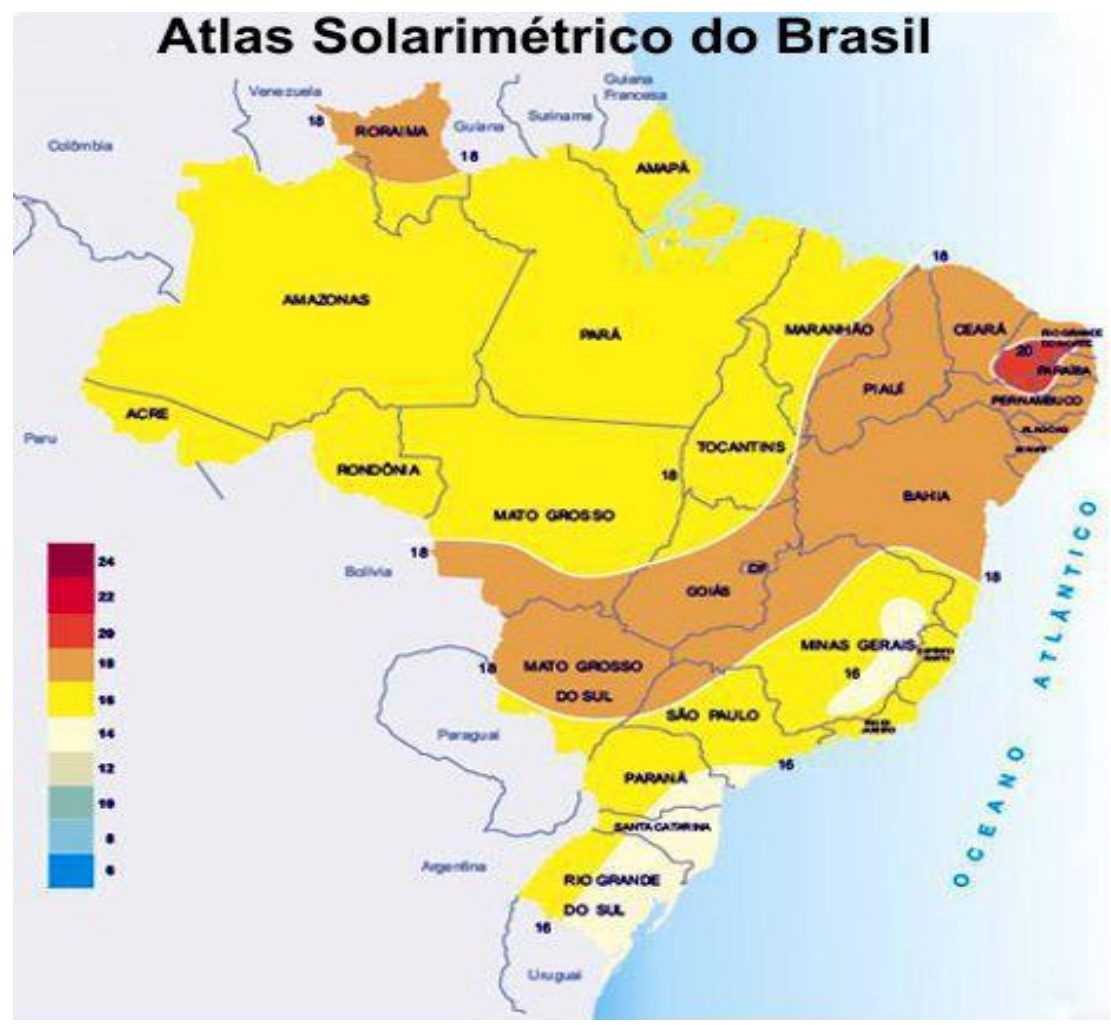

Figura 1: Atlas Solarimétrico Brasileiro. (TIBA, C. et al, 2000).

O Estado do Ceará, mais recentemente teve a instalação da primeira usina de painéis fotovoltaicos em escala comercial no Brasil. Esta usina foi instalada na cidade de Tauá distante de aproximadamente $344 \mathrm{~km}$ da capital do Estado do Ceará. A capacidade inicial de gerar energia elétrica fica em torno de $1 \mathrm{MW}$ (mega-watt), suficiente para abastecer 1,5 mil famílias. Existe uma grande expectativa de que essa usina possa em pouco tempo ser expandida sua capacidade de geração de energia elétrica da ordem de $50 \mathrm{MW}$. 
O resultado dos Atlas mostrado podem ser entendidos como ilustrações que padronizam as regiões de estudo, ou seja, os Estados que compõem o território Brasileiro. Mas além dessa padronização poderá ser necessário conhecer o Potencial Solar de uma localidade especifica e também tentar garantir que seus valores encontrados sofram poucas variações futuramente.

Com esse intuito uma forma de tentarmos garantir valores de radiação solar para geração de energia elétrica, com a finalidade de utilização em escala comercial poderá aparecer com pesquisas em previsibilidade de radiação solar, ou seja, buscar métodos que possam prever valores. $\mathrm{O}$ fato de fornecermos garantias sobre o quanto de energia elétrica poderá ser gerada a partir da radiação solar, principalmente em curto prazo, certamente trará mais segurança a muitos investidores.

O presente trabalho tem como objetivo utilizar técnicas estatísticas (médias móveis e projeção exponencial). Essas técnicas serão usadas para previsão de radiação solar incidente, com base em dados medidos na Universidade Federal do Ceará (UFC), campus Fortaleza, cedidos pelo Laboratório de Energias Alternativas (LEA). Esse estudo poderá auxiliar na tentativa de descobrir métodos satisfatórios que busquem responder, o quanto de energia elétrica, poderá ser gerada a partir da previsão de valores da radiação solar encontrada.

\section{MATERIAL E MÉTODOS}

Os dados de radiação solar incidente, coletados para a realização do presente estudo foram medidos com um piranômetro (instrumento para medir a irradiação solar sobre uma superfície plana. Em outras palavras, é um sensor desenhado para medir a densidade do fluxo de radiação solar $\mathrm{W} / \mathrm{m}^{2}$ num campo de 180 graus), nas dependências do Departamento de Engenharia Elétrica (DEE), da Universidade Federal do Ceará (UFC), campus Fortaleza, de propriedade do Laboratorio de Energias Alternativas - LEA da UFC. Esses dados são constituídos de médias registradas a cada dez minutos.

O presente trabalho tem como objetivo utilizar técnicas estatísticas (médias móveis e projeção exponencial). Essas técnicas serão usadas para previsão de radiação solar incidente, com base em dados medidos na Universidade Federal do Ceará (UFC), campus Fortaleza, no nordeste brasileiro. Esse estudo poderá auxiliar na tentativa de descobrir métodos satisfatórios que busquem responder, o quanto de energia elétrica, poderá ser gerada a partir da previsão de valores da radiação solar encontrada.

Os meses trabalhos nesse estudo foram março, abril, maio, outubro, novembro e dezembro, todos relativos ao ano de 2004. Vale ressaltar que de acordo com a Fundação Cearense de Meteorologia e Recursos Hídricos (FUNCEME), que diariamente faz o monitoramento do tempo em todo o estado, identificando desta forma a existência de praticamente duas estações sazonais que influenciam diretamente no tempo, ou seja, a estação chuvosa e a seca. Os meses de fevereiro a maio são considerados períodos da estação chuvosa, também chamados de quadra chuvosa. Já os meses de setembro a dezembro são considerados períodos da estação seca.

Para a previsão de uma série temporal de radiação solar incidente em nossa região de estudo, foram utilizados os seguintes métodos estatísticos: médias móveis e projeção exponencial. 


\section{FORMULAÇÃO MATEMÁTICA}

O modelo matemático de Médias Móveis (comumente simbolizada por MA, sendo que essa abreviatura é do inglês Moving Average), é constituído de médias aritméticas baseadas em dados passados de uma determinada série temporal. Ou seja, utilizam como previsão para um determinado período no futuro a média das observações passadas. As (MA) podem ser simples, centradas ou ponderadas. Para nosso estudo, ou seja, previsão de radiação solar incidente será utilizado o modelo de média móvel simples que conforme (GONÇALVES, F. 2007), matematicamente é representado por

$$
F_{\mathrm{t}+1}=\frac{1}{n} \sum_{\mathrm{j}=1}^{n} A_{t-\mathrm{j}+1},
$$

onde:

n (é o número total de observações), $A_{j}$ (valor observado no período j) e $F_{j}$ (valor projetado para o período j).

Na eq. (1), n (também pode ser chamado de janela de observações) pode ser considerado como um parâmetro a ser ajustado. Segundo Gonçalves F. (2007), se a série temporal em estudo apresentar muita aleatoriedade em seus padrões um número maior de observações podem ser utilizadas no cálculo da média móvel, ou seja, podemos dizer que a média móvel neste caso fica mais imune a ruídos e movimentos curtos. Já para o caso de séries que apresentam poucas flutuações aleatórias nos dados ou mudança significativa, um número menor deve ser usado para o tamanho da janela de observações, pois caso contrário a série prevista poderá reagir de maneira muito lenta a estas mudanças significativas.

A Projeção Exponencial Simples (PES) também é chamada de Suavização Exponencial (SE), está técnica tenta prever valores futuros de uma série temporal baseado em uma série temporal passada. De acordo com (HYNDMAN, R. J., KOEHLER, A. B., ORD, j, k., SNYDER, R. D. 2008) sua representação matemática é dada na eq. (2), abaixo:

$$
\bar{y}_{T}=\bar{y}_{T-1}+\alpha\left(y_{T}-\bar{y}_{T-1}\right),
$$

onde: $\mathrm{y}_{T}=$ representa a previsão para o tempo seguinte, $\mathrm{y}_{T}=$ representa o dado atual da série temporal real, $\mathrm{y}_{\mathrm{T}-1}=$ representa a previsão do tempo anterior e $\boldsymbol{\alpha}=$ é chamado de constante de suavização, que por sua vez, varia entre $0 \leq \boldsymbol{\alpha} \leq 1$.

Para a utilização da eq. (2) é preciso que no início do processo de previsão seja escolhido um valor inicial para $\mathrm{y}_{\mathrm{T}}$ (geralmente utiliza-se o primeiro valor da série temporal real) e também um valor para a constante de suavização $\boldsymbol{\alpha}$. É importante dizer que valores de $\boldsymbol{\alpha}$ pequenos produzem previsões que dependem mais das observações passadas, pois o peso das mesmas será então menor. Enquanto que valores de $\alpha$ próximos de 1 dependem das observações mais recentes, que são observações que recebem o maior peso. A dificuldade de utilizarmos a eq. (2) para fazer previsão de séries temporais reside na escolha de $\boldsymbol{\alpha}$. Nesse trabalho a escolha deste parâmetro será feita baseada em estatística de erros (que será visto em seção posterior), quando falarmos em erro quadrático médio (comumente simbolizada por RMSE, sendo que essa abreviatura é do inglês Root Mean Squared Error), ou seja, procuramos um valor de $\alpha$ que nos ofereça o menor 
(RMSE) possível quando comparado a série temporal real com série temporal prevista na utilização da (PES).

Para que seja feita uma comparação entre as séries temporais reais e prevista, com a finalidade de identificarmos o grau de proximidade entre valores de ambas, será realizada uma análise estatística de erros. Através desta análise é possível observarmos se existe semelhança ou não, entre dados reais e previstos.

O Erro Sistemático (ou do inglês, bias, comumente simbolizado por $B E$ ), mede a tendência do modelo em superestimar ou subestimar uma variável, e é definido matematicamente por,

$$
\mathrm{BE}=\frac{1}{n} \sum_{\mathrm{i}=1}^{n}\left(\bar{y}_{i}-y_{i}\right) \text {, }
$$

onde: $y_{i}$ (representa valor individual da série temporal real), e $y_{i}$ (representa valor individual da série temporal prevista).

Um valor positivo de $B E$ indica uma predisposição do modelo em superestimar uma variável particular. Já um valor negativo, uma subestimação do modelo na avaliação de uma variável. Maiores detalhes sobre o erro de bias em (PEDRO, H.T.C., 2012; CAMELO, H.N., 2007).

O Erro Quadrático Médio (simbolizado por RMSE, sendo que essa abreviatura é do inglês Root Mean Squared Error) representa as diferenças individuais quadráticas entre as séries temporais reais e previstas, que pode ser definido matematicamente por,

$$
\mathrm{RMSE}=\sqrt{\frac{1}{n} \sum_{\mathrm{i}=1}^{n}\left(y_{i}-y_{i}\right)^{2}},
$$

Vale ressaltar que as variáveis BE e RMSE ambas representadas nas eqs. (3) e (4), possuem unidade de medida de acordo com a série temporal em estudo, ou seja, no nosso caso temos que sua unidade é dada em (Watt por metro quadrado, simbolizado por $\mathrm{W} / \mathrm{m}^{2}$ ). É importante ressaltar que o RMSE pode ainda ser interpretado da seguinte maneira: Se caso temos grandes valores desta variável, os mesmos representam grandes erros nas variáveis previstas, e valores próximos de zero indicam uma previsão quase perfeita. Maiores detalhes sobre o Erro Quadrático Médio podem ser obtidos em (PEDRO, H. T.C., 2012; CAMELO, H.N., 2007).

Outra forma de medida de erro é a Média do Erro Absoluto Percentual (simbolizado por $M A P E$, do iglês Mean Absolute Percentage Error). A grande vantagem de utilizarmos essa expressão está na sua representação em termos percentuais (\%) que fornece um rápido entendimento. Já uma desvantagem que deve ser considerada está no sentido de que se por acaso, o valor real for muito pequeno, qualquer discrepância faz o MAPE "explodir". A expressão utilizada com essa variável é representada por,

$$
\mathrm{MAPE}=\frac{1}{n} \sum_{\mathrm{i}=1}^{n}\left|\frac{\left(y_{i}-y_{i}\right)}{y_{i}}\right| \times 100 .
$$

Maiores detalhes sobre estatística de erros, em especial as eqs. (3), (4) e (5), que serão usadas nesse trabalho pode ser obtido em (MONTGOMERY, D. C.; JENNINGS, C. L.; KULAHCI, M. 2008). 


\section{RESULTADOS}

Na Tab. 1, é possível identificar valores correspondentes ao cálculo de estatística de erros para os meses de março, abril e maio. No tocante ao RMSE, ficou constatado que a previsão realizada pela Projeção Exponencial Simples (PES) oferece a melhor escolha em relação aos métodos de Médias Móveis (MA). Essa observação é baseada em um menor RMSE destacado na cor vermelha. Totalizando os três meses o RMSE varia de 0,72 a $271,58 \mathrm{~W} / \mathrm{m}^{2}$.

Tabela 1: Estatística de erros para os meses de março, abril e maio.

\begin{tabular}{|c|c|c|c|c|c|c|}
\hline \multirow{2}{*}{$\begin{array}{c}\text { Março } \\
\text { Hora }\end{array}$} & \multicolumn{2}{|c|}{ RMSE $\left(W / m^{2}\right)$} & \multicolumn{2}{|c|}{$\mathrm{BE}\left(\mathrm{W} / \mathrm{m}^{2}\right)$} & \multicolumn{2}{|c|}{ MAPE (\%) } \\
\hline & MA & PES & MA & PES & MA & PES \\
\hline 05:00 & 3,24 & 2,55 & 0,03 & 0,03 & 284,27 & 221,04 \\
\hline 06:00 & 22,48 & 18,64 & $-1,11$ & $-3,13$ & 159,78 & 146,87 \\
\hline 07:00 & 103,39 & 87,61 & $-5,41$ & $-18,60$ & 110,93 & 102,54 \\
\hline 08:00 & 201,93 & 163,51 & $-2,79$ & $-27,17$ & 114,58 & 101,22 \\
\hline 09:00 & 225,51 & 182,69 & $-9,92$ & $-32,91$ & 133,19 & 130,86 \\
\hline $10: 00$ & 310,69 & 247,81 & $-4,95$ & $-15,38$ & 93,19 & 82,59 \\
\hline $11: 00$ & 332,47 & 264,90 & $-14,90$ & $-52,58$ & 62,50 & 51,45 \\
\hline $12: 00$ & 319,04 & 271,58 & $-16,80$ & $-34,31$ & 78,47 & 74,58 \\
\hline $13: 00$ & 272,28 & 250,98 & $-6,95$ & $-5,27$ & 75,58 & 76,63 \\
\hline $14: 00$ & 207,22 & 186,18 & $-5,88$ & $-14,94$ & 50,84 & 52,56 \\
\hline $15: 00$ & 148,46 & 136,12 & $-4,13$ & 0,45 & 46,04 & 48,50 \\
\hline $16: 00$ & 88,64 & 80,47 & $-0,42$ & 0,91 & 51,37 & 52,66 \\
\hline $17: 00$ & 8,98 & 7,25 & $-0,16$ & $-0,13$ & 40,90 & 32,16 \\
\hline Abril & MA & PES & MA & PES & MA & PES \\
\hline 05:00 & 0,76 & 0,72 & 0,03 & $-0,08$ & 24,04 & 23,51 \\
\hline 06:00 & 27,18 & 23,75 & $-0,36$ & $-1,59$ & 46,82 & 41,40 \\
\hline 07:00 & 109,22 & 99,25 & 3,40 & 5,73 & 58,16 & 60,14 \\
\hline 08:00 & 180,18 & 154,86 & $-2,79$ & $-27,17$ & 74,47 & 68,02 \\
\hline 09:00 & 196,13 & 188,26 & $-9,92$ & $-32,91$ & 45,36 & 45,42 \\
\hline $10: 00$ & 231,65 & 207,52 & $-4,95$ & $-15,38$ & 41,02 & 44,24 \\
\hline $11: 00$ & 293,60 & 240,67 & $-14,90$ & $-52,58$ & 46,57 & 40,75 \\
\hline $12: 00$ & 236,36 & 328,18 & $-16,80$ & $-34,31$ & 33,69 & 38,16 \\
\hline $13: 00$ & 230,79 & 196,08 & $-6,95$ & $-5,27$ & 71,93 & 69,49 \\
\hline $14: 00$ & 205,61 & 179,29 & $-5,88$ & $-14,94$ & 60,43 & 63,89 \\
\hline $15: 00$ & 130,08 & 114,37 & $-4,13$ & 0,45 & 45,57 & 44,41 \\
\hline $16: 00$ & 75,36 & 61,78 & $-0,42$ & 0,91 & 56,24 & 49,31 \\
\hline $17: 00$ & 5,74 & 5,50 & $-0,16$ & $-0,13$ & 33,81 & 34,35 \\
\hline Maio & MA & PES & MA & PES & MA & PES \\
\hline 05:00 & 1,32 & 1,23 & $-0,05$ & $-0,03$ & 32,87 & 30,72 \\
\hline 06:00 & 33,52 & 28,13 & $-2,19$ & 4,20 & 40,08 & 38,38 \\
\hline
\end{tabular}




\begin{tabular}{|c|c|c|c|c|c|c|}
\hline $07: 00$ & 94,76 & 84,82 & $-3,27$ & 14,64 & 34,12 & 33,40 \\
\hline $08: 00$ & 103,37 & 83,45 & $-9,14$ & $-19,28$ & 18,29 & 15,11 \\
\hline $09: 00$ & 172,42 & 143,78 & $-18,11$ & $-22,88$ & 26,22 & 23,11 \\
\hline $10: 00$ & 235,88 & 203,60 & 9,48 & 42,91 & 35,30 & 35,52 \\
\hline $11: 00$ & 233,73 & 218,95 & 12,92 & 19,83 & 34,40 & 32,76 \\
\hline $12: 00$ & 218,51 & 196,93 & 18,84 & 40,10 & 29,91 & 30,02 \\
\hline $13: 00$ & 167,04 & 159,34 & 7,24 & 8,23 & 24,15 & 23,72 \\
\hline $14: 00$ & 142,86 & 134,99 & 6,15 & 6,66 & 23,39 & 23,70 \\
\hline $15: 00$ & 121,98 & 119,64 & 9,05 & 2,29 & 19,81 & 26,49 \\
\hline $16: 00$ & 63,73 & 53,62 & 4,08 & 12,11 & 40,91 & 39,88 \\
\hline $17: 00$ & 3,67 & 2,80 & 0,37 & 0,04 & 84,85 & 62,57 \\
\hline
\end{tabular}

No tocante a variável MAPE, também é possível identificar que os menores valores são observados na previsão com a PES na maioria dos meses. Mas é importante ressaltar que como a MAPE mede o erro percentual na comparação entre duas séries temporais (no nosso caso, real e prevista), foi identificado valores absurdos, como por exemplo, 221,04 \% (maior valor da comparação, relativo às $05: 00 \mathrm{~h}$ do mês de março de 2004). No entanto, é possível identificar valores menores, como por exemplo, 15,11 \% (menor valor da comparação, relativo às 08:00 $\mathrm{h}$ do mês de maio de 2004). Uma tentativa de justificar grandes valores de RMSE e MAPE pode ser dada em função de estarmos falando em meses relativo à quadra chuvosa no Estado do Ceará já comentado na introdução, e, portanto, temos grandes variações de medição da radiação solar incidente, mas futuramente essa afirmação poderá ser investigada.

Ainda na Tab. 1, analisando a variável BE (onde como foi mencionado, seu valor positivo indica uma predisposição do modelo em superestimar uma variável particular. Já um valor negativo, uma subestimação do modelo na avaliação de uma variável), nesse contexto, a previsão com PE subestima os dados reais em uma variação entre $-52,58$ e $-0,03 \mathrm{~W} / \mathrm{m}^{2}$. Agora em termos de superestimação a previsão com PE varia entre 0,03 e 42,91 W/ $/ \mathrm{m}^{2}$.

Na Tab. 2, temos os resultados para a estatística de erros para os meses considerados do período seco no Estado do Ceará, ou seja, outubro, novembro e dezembro. Da mesma forma do período chuvoso (março, abril e maio), é possível afirmar que o RMSE em mais de $90 \%$ possui os menores valores para a previsão realizada com a variável PES. Esse resultado também pode ser afirmado para a variável MAPE. O RMSE varia entre 1,40 e 148,00 W/m². Já para o MAPE a variação encontra-se entre 4,48 (menor valor da comparação, relativo às 13:00 h do mês de outubro de 2004) e 26,47 \% (maior valor da comparação, relativo às 08:00 h do mês de dezembro de 2004). Esses resultados quando comparado com os obtidos pela Tab. 1, é possível concluir que no período seco utilizado o valor máximo de erro das variáveis RMSE e MAPE, são menores do que os observados no período chuvoso utilizado. 
Tabela 2: Estatística de erros para os meses de outubro, novembro e dezembro.

\begin{tabular}{|c|c|c|c|c|c|c|}
\hline \multirow{2}{*}{$\begin{array}{c}\text { Outubro } \\
\text { Hora }\end{array}$} & \multicolumn{2}{|c|}{ RMSE (W/m²) } & \multicolumn{2}{|c|}{$\mathrm{BE}\left(\mathrm{W} / \mathrm{m}^{2}\right)$} & \multicolumn{2}{|c|}{ MAPE (\%) } \\
\hline & MA & PES & MA & PES & MA & PES \\
\hline 05:00 & 3,19 & 2,99 & $-0,31$ & $-0,83$ & 21,97 & 16,67 \\
\hline 06:00 & 19,46 & 17,32 & $-1,87$ & $-4,70$ & 23,39 & 20,32 \\
\hline $07: 00$ & 72,75 & 61,08 & 2,68 & 6,40 & 26,01 & 22,06 \\
\hline 08:00 & 81,29 & 74,03 & 0,02 & $-0,30$ & 13,23 & 11,47 \\
\hline 09:00 & 133,41 & 111,66 & $-9,61$ & 17,70 & 17,44 & 15,23 \\
\hline $10: 00$ & 105,58 & 90,06 & 4,53 & 2,73 & 10,21 & 9,23 \\
\hline $11: 00$ & 87,28 & 77,44 & 2,20 & 15,01 & 7,98 & 6,69 \\
\hline $12: 00$ & 87,29 & 81,92 & 0,77 & 7,24 & 8,19 & 7,34 \\
\hline $13: 00$ & 51,17 & 48,98 & 0,95 & 0,90 & 4,95 & 4,48 \\
\hline $14: 00$ & 57,23 & 50,37 & 0,85 & 8,21 & 5,97 & 4,65 \\
\hline $15: 00$ & 35,22 & 33,42 & 1,46 & 4,49 & 5,99 & 5,81 \\
\hline $16: 00$ & 17,32 & 16,41 & 6,38 & 5,57 & 13,71 & 12,48 \\
\hline $17: 00$ & 1,52 & 1,40 & 0,22 & 0,41 & 16,15 & 15,84 \\
\hline Novembro & MA & PES & MA & PES & MA & PES \\
\hline 05:00 & 2,86 & 2,39 & 0,06 & $-0,29$ & 16,42 & 13,63 \\
\hline $06: 00$ & 15,92 & 15,01 & $-0,73$ & $-0,66$ & 18,69 & 17,46 \\
\hline 07:00 & 54,97 & 47,05 & 2,32 & 8,35 & 20,70 & 17,90 \\
\hline $08: 00$ & 124,68 & 114,54 & 4,42 & 19,36 & 22,79 & 21,73 \\
\hline 09:00 & 135,48 & 124,34 & 17,82 & 38,32 & 19,47 & 18,52 \\
\hline $10: 00$ & 113,40 & 98,50 & 8,31 & 14,71 & 12,56 & 11,10 \\
\hline $11: 00$ & 80,63 & 71,37 & 3,72 & 13,28 & 7,53 & 6,32 \\
\hline $12: 00$ & 62,86 & 58,94 & 4,49 & 10,57 & 5,40 & 5,21 \\
\hline $13: 00$ & 76,97 & 66,59 & 5,53 & 14,51 & 8,06 & 6,34 \\
\hline $14: 00$ & 54,50 & 47,65 & 0,13 & 4,22 & 6,16 & 5,89 \\
\hline $15: 00$ & 51,89 & 44,90 & 3,07 & 10,79 & 9,32 & 7,51 \\
\hline $16: 00$ & 8,37 & 8,12 & $-0,61$ & $-0,73$ & 6,72 & 6,07 \\
\hline $17: 00$ & 2,16 & 1,92 & $-0,07$ & $-0,20$ & 22,57 & 18,30 \\
\hline Dezembro & MA & PES & MA & PE & MA & PES \\
\hline 05:00 & 3,11 & 2,79 & 0,38 & 0,73 & 37,48 & 40,17 \\
\hline $06: 00$ & 16,28 & 15,18 & 1,16 & 2,11 & 24,90 & 23,47 \\
\hline 07:00 & 46,58 & 41,59 & 2,16 & 11,41 & 21,21 & 20,80 \\
\hline 08:00 & 129,18 & 114,37 & $-4,73$ & 15,55 & 29,63 & 26,57 \\
\hline 09:00 & 158,01 & 143,81 & 5,34 & 30,25 & 28,29 & 27,75 \\
\hline $10: 00$ & 181,86 & 148,00 & $-0,79$ & $-26,04$ & 27,55 & 23,00 \\
\hline $11: 00$ & 113,47 & 94,28 & 3,19 & 22,73 & 12,39 & 10,54 \\
\hline $12: 00$ & 146,14 & 115,87 & 1,65 & 26,45 & 16,00 & 12,72 \\
\hline
\end{tabular}




\begin{tabular}{|c|c|c|c|c|c|c|}
\hline $13: 00$ & 117,35 & 103,65 & $-2,97$ & 16,74 & 12,99 & 11,92 \\
\hline $14: 00$ & 119,84 & 100,03 & $-1,19$ & 19,57 & 17,86 & 14,64 \\
\hline $15: 00$ & 79,92 & 69,75 & $-3,80$ & $-2,45$ & 17,81 & 17,67 \\
\hline $16: 00$ & 27,04 & 22,85 & $-3,30$ & $-6,52$ & 15,52 & 14,96 \\
\hline $17: 00$ & 3,07 & 2,92 & $-0,33$ & $-0,74$ & 15,46 & 13,45 \\
\hline
\end{tabular}

Ainda na Tab. 2, analisando a variável $\mathrm{BE}$, a previsão com PE subestima os dados reais em uma variação entre $-26,04 \mathrm{e}-0,20 \mathrm{~W} / \mathrm{m}^{2}$. Agora em termos de superestimação a previsão com $P E$ varia entre 0,41 e $38,32 \mathrm{~W} / \mathrm{m}^{2}$. Esses resultados quando comparados com os da Tab. 2, revelam que no período seco utilizando a previsão com $\mathrm{PE}$, tanto subestima como superestima os dados reais com menores valores em relação ao período chuvoso utilizado.

Uma vez que a previsão com o método de Projeção Exponencial tenha apresentado em sua maioria menores valores de erros do tipo RMSE e MAPE, quando comparado com o método de previsão de Médias Móveis, a partir dessa informação os próximos resultados serão mostrados com foco na Projeção Exponencial.

Na Fig. (2), temos a apresentação gráfica da média horária da Radiação Solar Incidente para o dia 30/10/2004. Em linha continua (na cor preta) é possível observar o perfil dos dados reais durante o dia e constatar que a média mais intensa foi as 11:00h com aproximadamente $950 \mathrm{~W} / \mathrm{m}^{2}$. Nesse mesmo horário a previsão em linha continua (na cor cinza claro) ficou com o valor de aproximadamente $900 \mathrm{~W} / \mathrm{m}^{2}$. Nesse caso específico foi identificado uma subestimação do modelo de previsão de aproximadamente $50 \mathrm{~W} / \mathrm{m}^{2}$. É possível também observar que que boa parte dos dados previstos pela projeção exponencial conseguem acompanhar os dados reais, principalmente entre os intervalos de 05:00h a 08:00h e 13:00h a 17:00h.

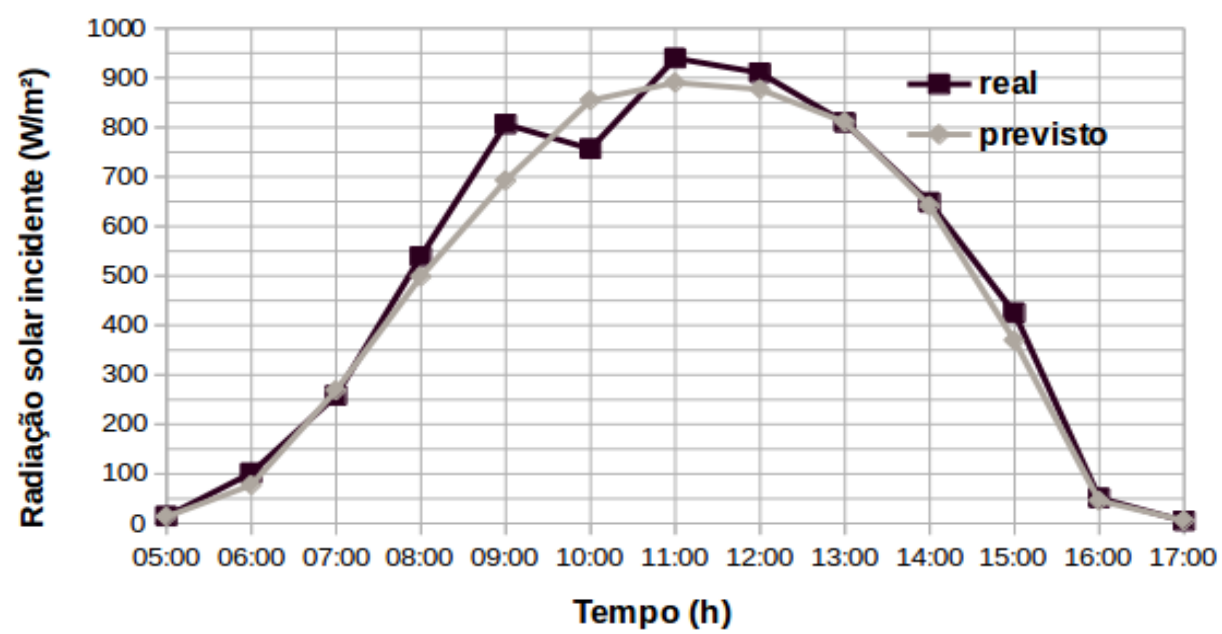

Figura 2. Comparação da Radiação Solar Incidente entre real e previsão para o dia 30/10/2004.

Na Fig. (3), temos a apresentação gráfica da média horária da Radiação Solar Incidente para o dia 30/11/2004. Analisando o valor máximo da média da radiação solar incidente, é possível identificar que o horário de maior intensidade é também as 11:00h. O valor real é de aproximadamente $1000 \mathrm{~W} / \mathrm{m}^{2}$. Agora para o modelo de previsão com projeção exponencial seu valor é de aproximadamente $900 \mathrm{~W} / \mathrm{m}^{2}$. Nesse caso, o modelo também subestimou o dado real. 
Entre os horários de 14:00h e 17:00h, a projeção exponencial se assemelham bastante com a série real. Exemplo disso, podemos destacar especificamente o horário de 16:00h em que as duas séries possuem valor de aproximadamente $100 \mathrm{~W} / \mathrm{m}^{2}$.

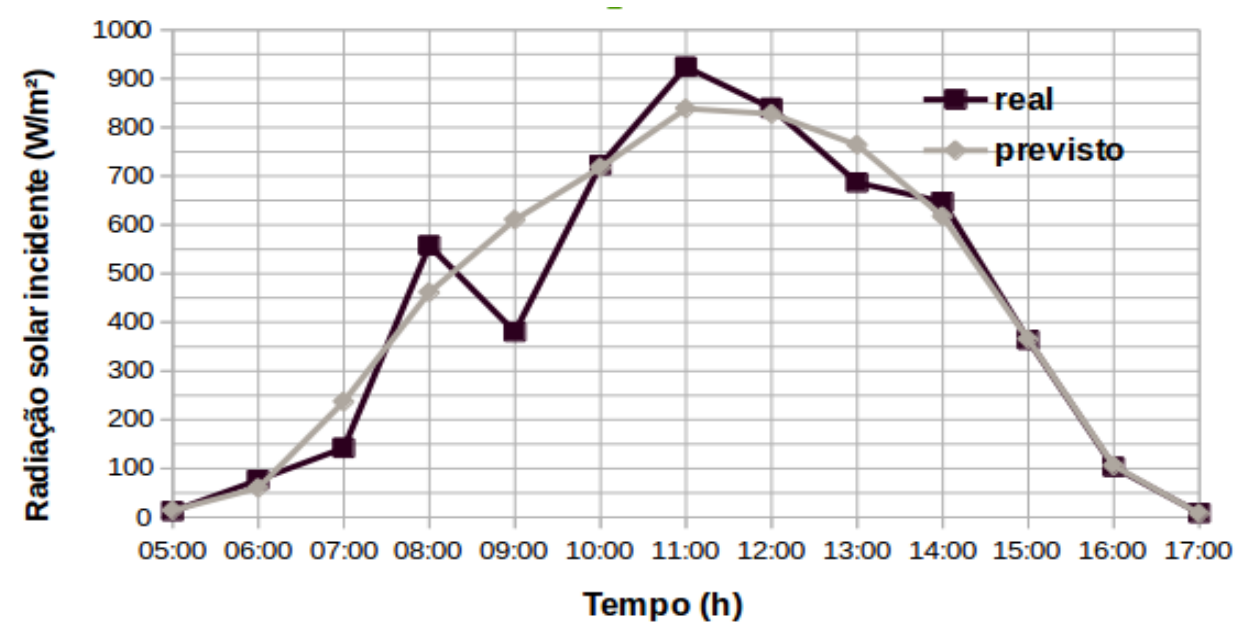

Figura 3. Comparação da Radiação Solar Incidente entre real e previsto para o dia 30/11/2004.

Na Fig. (4), temos a representação gráfica da média horária da Radiação Solar Incidente para o dia 31/12/2004. A média de maior intensidade da Radiação Solar Incidente para a série temporal real, possui valor de aproximadamente $850 \mathrm{~W} / \mathrm{m}^{2}$ no horário de 12:00h. Já a previsão para o mesmo horário possui valor de aproximadamente $750 \mathrm{~W} / \mathrm{m}^{2}$.

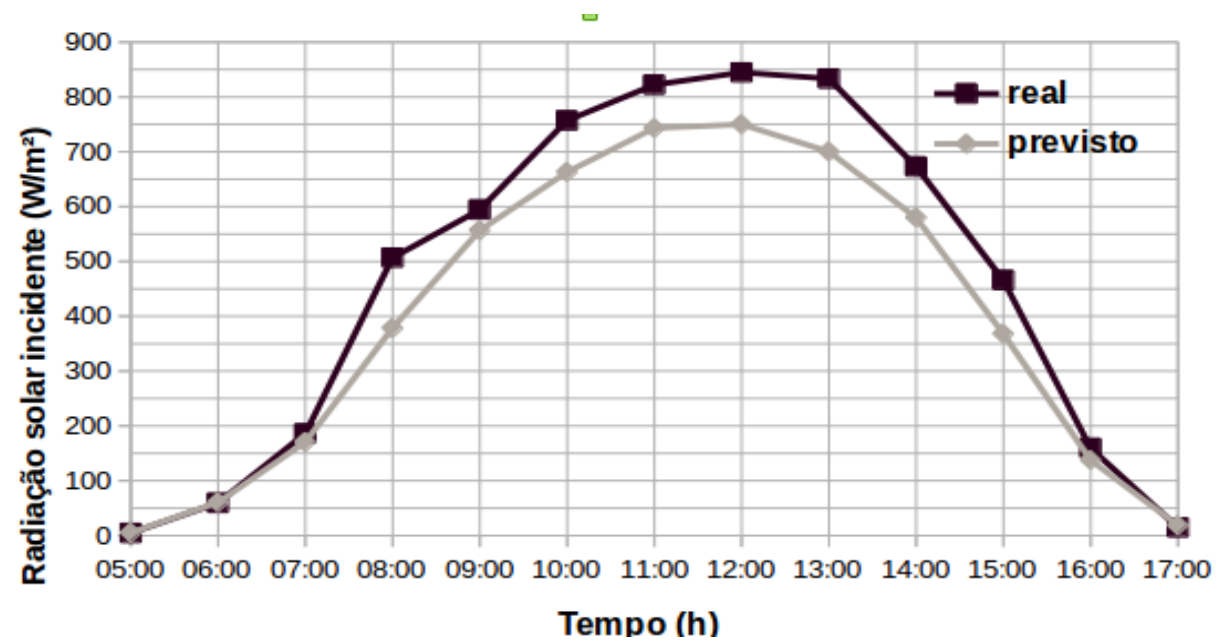

Figura 4. Comparação da Radiação Solar Incidente entre real e previsto para o dia 31/12/2004.

Na Fig. (5), temos a apresentação gráfica da média horária da Radiação Solar Incidente para o dia 31/03/2004. No horário de 11:00h, a média da Radiação Solar Incidente possui valor de pico de aproximadamente $980 \mathrm{~W} / \mathrm{m}^{2}$. A previsão para esse mesmo horário foi subestimada no valor de aproximadamente $740 \mathrm{~W} / \mathrm{m}^{2}$. De forma semelhante aos resultados das figuras anteriores, pode ser observado que nos horários de final do dia, ou seja, entre 15:00h e 17:00h as duas séries temporais são bastante similares. 


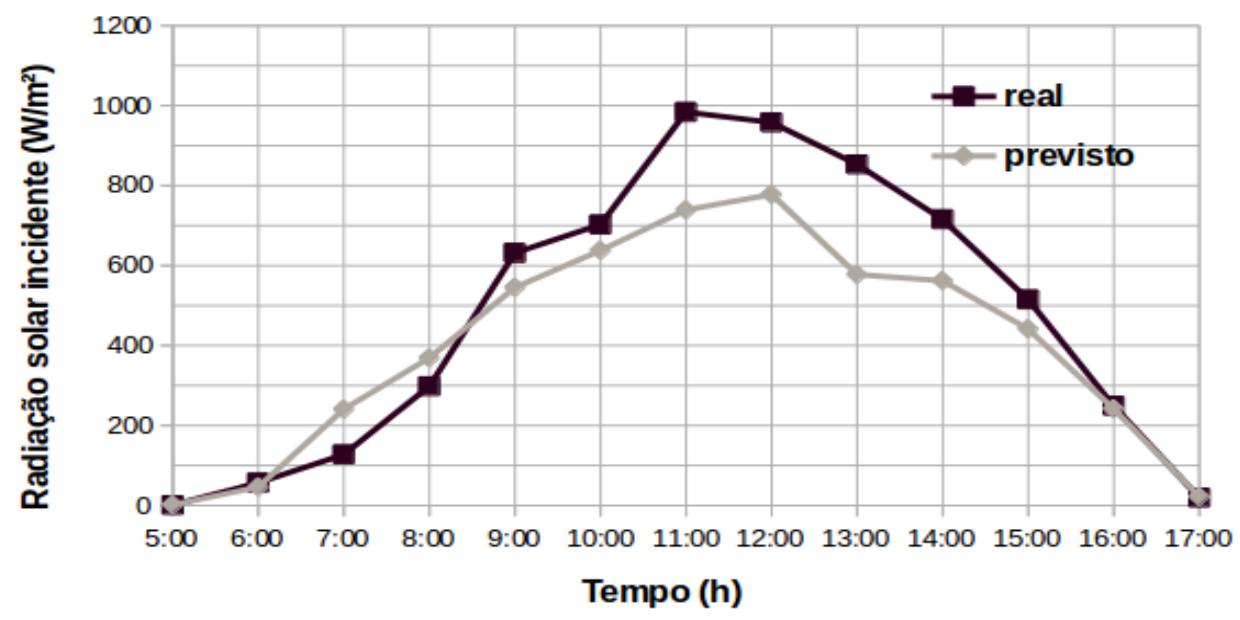

Figura 5. Comparação da Radiação Solar Incidente entre real e previsto para o dia 31/03/2004.

Na Fig. (6), temos a apresentação gráfica da média horária da Radiação Solar Incidente para o dia 31/04/2004. No horário de 10:00h, a média da Radiação Solar Incidente possui valor de pico de aproximadamente $900 \mathrm{~W} / \mathrm{m}^{2}$. A previsão para esse mesmo horário foi subestimada no valor de aproximadamente $600 \mathrm{~W} / \mathrm{m}^{2}$. Entre os horários de 05:00h e 08:00h, as duas séries se assemelham. Exemplo disso, podemos destacar especificamente o horário de 08:00h em que as duas séries possuem valor de aproximadamente $400 \mathrm{~W} / \mathrm{m}^{2}$.

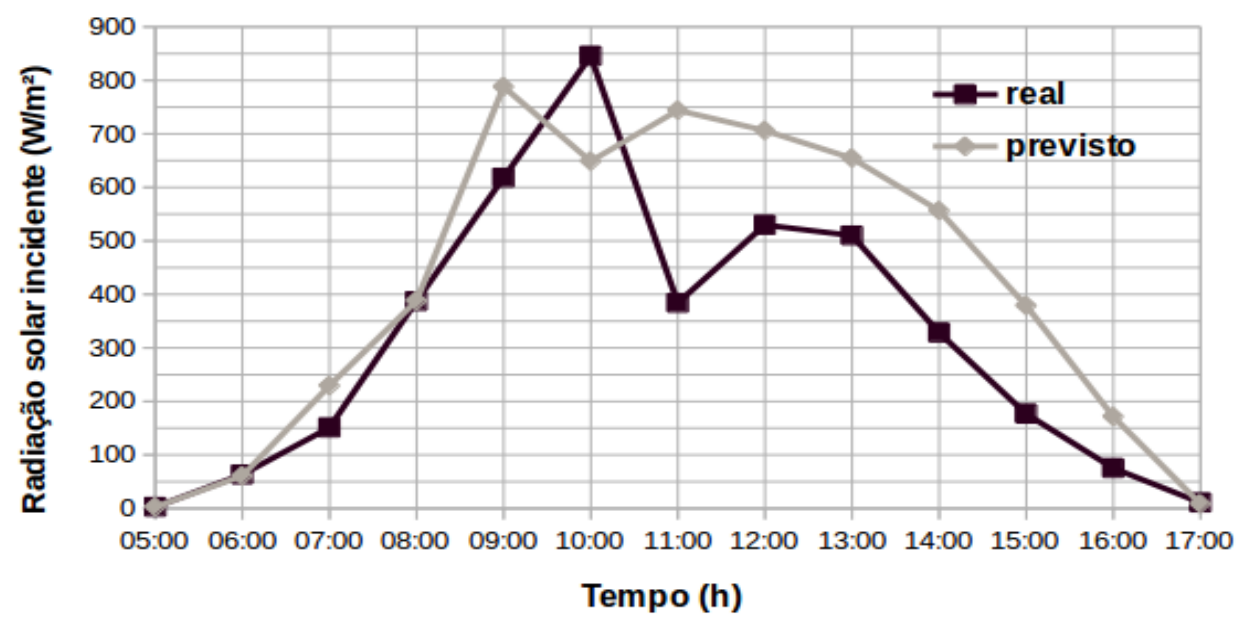

Figura 6. Comparação da Radiação Solar Incidente entre real e previsto para o dia 31/04/2004.

Na Fig. (7), temos a apresentação gráfica da média horária da Radiação Solar Incidente para o dia 31/05/2004. Entre os horários de 05:00h a 09:00h, é possível identificar semelhanças entre a série prevista e a série real, como por exemplo, as 08:00h ambas possuem valor próximo de 600 $\mathrm{W} / \mathrm{m}^{2}$. Ma vale ressaltar que a série prevista como um todo não acompanhou a real principalmente entre os horários de 11:00h até as 16:00h. 


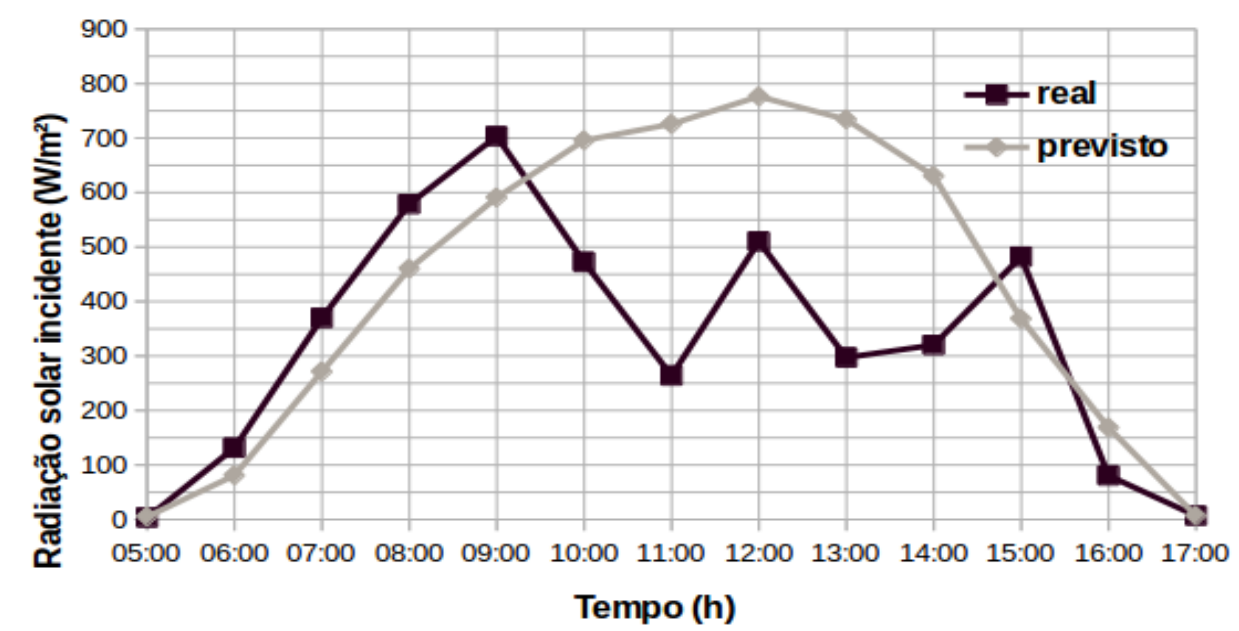

Figura 7. Comparação da Radiação Solar Incidente entre real e previsto para o dia 31/05/2004.

Fazendo uma comparação entre os períodos estudados, ou seja, chuvoso e seco, pode se afirmar que a previsão via método de projeção exponencial viabiliza melhores resultados no período seco. Isto é baseado no fato de que esse método consegue acompanhar melhor ao longo do dia os dados reais, como representado nas Figs. (2), (3) e (4). Nessas figuras, sobretudo nos horários entre 13:00h e 17:00h o que se pode identificar é uma melhor comparação entre previsão e real em relação aos demais horários.

\section{CONCLUSÕES E PERSPECTIVAS FUTURAS}

Os mapas de potencial solar confeccionados até o presente momento no Brasil demonstram que as regiões do Nordeste, em especial no Estado do Ceará, possuem grande potencial a ser explorado. A partir dessa afirmativa, faz-se necessário avançar em pesquisas em geração solar aplicado a geração de energia elétrica. Dessa forma, é possível pensar que a diversificação de formas de energia em nossa matriz energética nos garantirá uma maior tranqüilidade, sobretudo em relação ao uso de eletricidade. Além disso, é sabido que a utilização de fontes renováveis é o principal meio difundido pela comunidade cientifica no sentido de amenizar impactos de poluentes atmosféricos e, portanto, uma maneira de alcançarmos o desenvolvimento sustentável.

O Estado do Ceará tem se notabilizado como a região do país que mais tem crescido nos últimos anos em exploração de seu regime de ventos para geração de energia elétrica. O Ceará é considerado o pioneiro em instalação de parques eólicos em dunas, e atualmente possui aproximadamente 1,2 GW de capacidade de potência eólica instalada, segundo a Associação Brasileira de Energia Eólica - ABEEólica em 2014. Em termos de geração solar, o Ceará também é considerado pioneiro, só que agora em relação a ser a primeira usina solar de forma comercial instalada no Brasil e em plena operação. Trata-se da usina solar da cidade deTauá, com geração de energia elétrica através de painéis fotovoltaicos da ordem de $1 \mathrm{MW}$, capaz de atender a 1,5 mil famílias. Evidentemente que esse valor de geração solar ainda é muito pequeno em relação à geração eólica.

Através da utilização dos métodos estatísticos de previsão (médias móveis e projeção exponencial) para geração de séries temporais, aplicados a radiação solar incidente, cujos dados reais foram medidos nas dependências do Departamento de Engenharia Elétrica (DEE) da 
Universidade Federal do Ceará (UFC), campus Fortaleza, ficou constatado que o método de projeção exponencial é a melhor escolha para a previsão de série temporais. Essa conclusão é baseada no fato de que este método tanto para o período chuvoso, como para o período seco, fornece os menores valores de RMSE como por exemplo, para o mês de outubro no horário de 17:00h temos $1,40 \mathrm{~W} / \mathrm{m}^{2}$, e para o MAPE, temos o valor de $4,48 \%$ também para o mês de outubro as 13:00h.

Posteriormente outros métodos de previsão de séries temporais poderão ser testados, com o objetivo de minimizar cada vez mais, erros na comparação com séries observadas de radiação solar incidente para nossa região de estudo. Métodos estatísticos como o modelo ARIMA, métodos de simulação computacional, através do uso de modelos atmosféricos regionais de mesoescala como, por exemplo, o WRF, e também a utilização de outros métodos computacionais como é o caso das redes neurais artificiais. Esse trabalho tem como objetivo incentivar o uso da fonte solar para geração de energia elétrica no Estado do Ceará, nordeste Brasileiro.

\section{REFERÊNCIAS BIBLIOGRÁFICAS}

1. ABEEólica - Associação Brasileira de Energia Eólica. Disponível em <http://www.portalabeeoli ca.org.br/>. Acesso em: 22 de jan. de 2015.

2. CAMELO, H. N. Estudo Numerico do Vento Aracati para Caracterização de seu Potencial Eólico. 2006. 95 f. Dissertação (Mestrado em Ciências Físicas Aplicadas) - Universidade Estadual do Ceará, Fortaleza 2007.

3. GONÇALVES, F. Excel avançado 2003/2007 - Analise de Previsão de Demanda. 1 ed. Rio de Janeiro: Editora Ciência Moderna, 2007.

4. HYNDMAN, R.J., KOEHLER, A.B., ORD, J.K., SNYDER, R.D. Forecasting with Exponential Smoothing - The State Space Approach. 1 ed. New Jersey: Editora Springer, 2008.

5. MONTGOMERY, D. C., C. L. JENNINGS, and M. KULAHCI, Introduction to Time Series Analysis and Forecasting. 6 ed. New York: Wiley-Interscience, 2008.

6. PEDRO, H.T.C., C.F.M. Assessment of forecasting techniques for solar power production with no exogenous inputs. Solar Energy, v. 86, p. 2017-2028, mai. 2012.

7. TIBA, C. et al. Atlas Solarimétrico do Brasil: banco de dados terrestres. Recife: Editora Universitária da Universidadade Federal de Pernambuco - UFPE, 2000. 